\title{
De l'âme première à l'autonomie seconde : la cosmologie politique des Lois
}

From the First Soul to the Second Autonomy: the Political Cosmology of the Laws

\section{Fulcran Teisserenc}

\section{(2) OpenEdition}

1 Journals

Édition électronique

URL : https://journals.openedition.org/cps/1455

DOI : $10.4000 /$ cps. 1455

ISSN : 2648-6334

Éditeur

Presses universitaires de Strasbourg

\section{Édition imprimée}

Date de publication : 30 mai 2019

Pagination : $39-70$

ISBN : 979-10-344-0047-8

ISSN : $1254-5740$

Référence électronique

Fulcran Teisserenc, «De l'âme première à l'autonomie seconde : la cosmologie politique des Lois », Les Cahiers philosophiques de Strasbourg [En ligne], 45 | 2019, mis en ligne le 30 mai 2019, consulté le 11 novembre 2022. URL : http://journals.openedition.org/cps/1455; DOI : https://doi.org/10.4000/cps 1455

\section{(c) (i) (8)}

Creative Commons - Attribution - Pas d'Utilisation Commerciale - Partage dans les Mêmes Conditions 4.0 International - CC BY-NC-SA 4.0

https://creativecommons.org/licenses/by-nc-sa/4.0/ 


\section{De l'âme première à l'autonomie seconde: la cosmologie politique des Lois}

Fulcran Teisserenc

La dernière cosmologie de Platon s'inscrit dans le cadre de la lutte contre les impies. Ces derniers se divisent en trois catégories. Les premiers nient purement et simplement l'existence des dieux. Les seconds l'admettent, mais considèrent qu'ils sont indifférents au sort des hommes. Les troisièmes ne sont pas coupables des deux premières erreurs, mais estiment que les dieux sont corruptibles et que leurs bienfaits s'achètent moyennant prières et offrandes. C'est pour contrer les athées proprement dits (la première espèce d'impies) que l'Étranger d'Athènes développe une argumentation en faveur de l'existence des dieux, tandis qu'une plus exacte conception de la providence divine permet de corriger les égarements des autres impies.

Les croyances irréligieuses sont supposées par l'Athénien être à la source des conduites les plus dangereuses pour la société (elles causent selon lui non seulement les actions impies, mais encore toutes les paroles contraires à la loi $\left.{ }^{1}\right)$. Combattre de telles croyances, et surtout empêcher leur formation même dans l'esprit des habitants de la cité des Magnètes, tel est donc le premier des soucis du législateur. D'où la place éminente que va tenir, dans le corpus de lois à destination de la future colonie de Crète, cette réfutation des athées. Le prototype des preuves de l'existence de Dieu est ainsi élaboré dans une perspective politique, afin d'affermir les convictions indispensables à l'harmonie de la vie sociale.

Cette "démonstration" reprend certains éléments de la psychologie et de la cosmologie du Phèdre. Il ne s'agit pourtant pas, en dépit $\mathrm{du}$ caractère argumentatif du passage et de l'absence de narration

1 Platon, Lois, X 885b5.

Les Cahiers Philosophiques de Strasbourg, i / 2019 
mythologique, d'une preuve au sens strict. L'Athénien sait bien qu'il peut seulement, en l'occurrence, persuader du vraisemblable, non pas administrer la démonstration d'un gouvernement divin du cosmos. Deux éléments fameux dans cette discussion, l'automotricité de l'âme et la circularité du mouvement cosmique, se distinguent en réalité par leur absence de contribution directe à la manifestation de l'existence des dieux. Le premier apparaît au final superflu, le second ne fournit tout au plus qu'un indice, et non une preuve.

Mon propos dans cet article est d'abord d'étayer ce double point. Il est ensuite d'expliquer malgré tout la présence de ces thèses dans l'argumentaire platonicien. Je souhaite établir que leur inutilité théologique, dont Platon devait être parfaitement conscient, est de nature à faire ressortir par contraste leur utilité "cosmopolitique»: elles participent à la fabrication d'une image du monde qui facilite l'administration de la cité, dans la mesure où elles contribuent à faire $\mathrm{du}$ monde un modèle formel du gouvernement de soi. L'intelligence, pour s'exercer pleinement à l'échelle humaine, a besoin de se donner un référent cosmique, et les Lois offrent une description vivante de cette élaboration du cadre naturel au sein duquel la liberté des hommes trouve sa justification et son orientation.

Quelle est la doctrine des athées que Platon entend combattre? Et s'agit-il d'un groupe constitué, développant une appréhension unifiée du réel, ou est-ce un groupe disparate, agrégé par un Platon reconstruisant leur propos de façon synthétique?

La question est difficile à trancher, faute de noms propres cités par l'Athénien. Il faut toutefois noter que dans tous les dialogues tardifs (en dehors du Timée) où Platon aborde les questions cosmologiques et élabore sa théologie, il évoque toujours un ensemble déterminé d'opposants, de gens habiles et retors (deinoi) qui développent des vues matérialistes ${ }^{2}$. Ce n'est donc pas une fantaisie des seules Lois.

D'autre part, les athées tels qu'ils sont évoqués par l'Athénien ne sont pas seulement matérialistes, ce sont avant tout des penseurs d'un devenir historique qui déploie une genèse progressive des formes de l'être. À la racine de la réalité, prennent place pour eux le hasard et la nature (889a5,

2 Platon, Phèdre, 245c2, Sophiste, 246b4 (cf. 265c8), Philèbe, 29a3. 
b1): combinés, ils donnent naissance aux premiers corps (les éléments), lesquels, par rencontre et agrégation des contraires, produisent les astres, la terre et le ciel tout entier et ce qu'il contient (889c). L'art vient bien après, et ses productions manquent de force et de consistance par rapport aux puissances naturelles dont elles sont l'émanation lointaine; parmi les ouvres de l'art, il faut compter les lois, les coutumes et les dieux (889e). Ces derniers se trouvent donc à la toute fin du processus, objets et produits d'une prescription législative. Ils ont donc autant d'existence que les conventions qui leur donnent naissance.

Or, voilà que des jeunes gens, exposés à ces thèses, renoncent à croire aux dieux tels que la loi les prescrit et en appellent à une vie selon la nature. Toutefois, cette conséquence qu'ils tirent de l'enseignement reçu (et à laquelle, manifestement, ils ne se tiendront pas, puisque l'athéisme est une maladie de la jeunesse que le temps guérit $\left.{ }^{3}\right)$, s'écarte en réalité de la doctrine professée par les maîtres, lesquels ne nient pas les dieux, mais les subordonnent à la loi (et en ce point, cet enseignement est exactement le même que celui qui est dispensé dans la cité des Magnètes $\left.^{4}\right)$. L'athéisme des jeunes gens découle plutôt de l'amplification de l'opposition de la nature à la loi - du primitif et de l'originel au fruit tardif de la civilisation -, et de la préférence accordée au premier terme sur le second. Cette amplification les amène à soutenir que, selon la nature, il n'y a pas de dieux, soit absolument, soit tels que la loi les détermine 5 .

La description par l'Athénien de cette théorie matérialiste ne fait pas état, dans un premier temps, de l'âme; celle-ci n'est introduite que dans l'énoncé de l'erreur de principe de cette théorie, qui réside dans l'inversion des priorités génétiques. Pour l'Athénien, et contrairement à ce que soutiennent les athées, l'âme est formée, ainsi que ce qui va avec, avant le corps ou les corps, lesquels doivent avoir été façonnés par l'art et l'âme. C'est donc à un renversement complet de la doctrine des "savants» que veut aboutir l'Athénien. Il ne peut le faire, pense-t-il, qu'en proposant une définition de l'âme, dont les termes sont, verrons-nous, quasiment identiques à ceux du Phèdre, mais ne mettent en évidence que sa seule priorité cinétique sur les autres corps. L'âme est davantage

3 Lois, X 888c2-3, cf. Sophiste, 265d1.

4 Voir F. Teisserenc, «Le dieu de la loi», p. 66.

5 Lois, X 890a5; il faut sous entendre un phusei dans l'explication de ce que pensent les jeunes gens athées. 
abordée comme un élément que comme une réalité individuée et sa nature propre ne doit rien à la rationalité, sans être exclusive de celle-ci. Nous constaterons d'ailleurs que seuls le nous (voũc) et son mouvement (ou considéré comme tel) tendent à favoriser la conviction que les dieux existent. Ils ne peuvent, toutefois, la rendre nécessaire.

\section{L'âme, première dans l'ordre de la génération (ou la liberté du commencement par soi-même)}

L'Athénien présente d'abord l'âme comme...

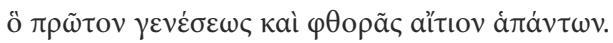

«La cause première de la génération et de la destruction de toute chose $»^{6}$.

Il précise ensuite:

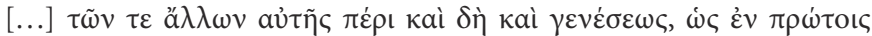

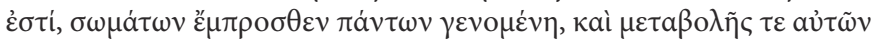

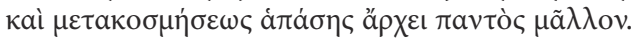

«[ils ont ignoré] entre autres choses au sujet précisément de sa génération, que l'âme est parmi les choses premières, née avant tous les corps, et qu'elle est plus que tout au principe de tout changement et de toute mise en ordre qui affectent ces corps ${ }^{7}$.

Ces deux textes ne sont pas rigoureusement synonymes: le premier fait de l'âme la cause universelle de toute naissance et de toute destruction, ce qui, sauf à supposer une régression à l'infini, semble impliquer qu'elle soit elle-même soustraite au devenir. Le second est plus restrictif, puisqu'il considère que l'âme est principe du changement dans les corps seulement et attribue à celle-ci une causalité positive: à la place de la phthora ( $\varphi \theta 0 \rho \dot{)})$, destruction, que mentionnait le premier, il fait figurer

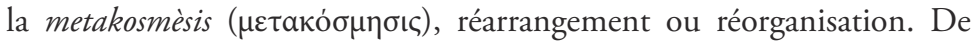
plus, et de manière quelque peu contradictoire avec ce qui précède, l'Athénien souligne le fait que l'âme elle-même est née, qu'elle a été l'objet d'une génération. Il serait certes loisible d'imaginer qu'elle ait pu être produite par une autre âme, mais sauf à régresser à nouveau à l'infini, il faut bien tomber sur une âme non engendrée. Or quand l'Athénien

6 Lois, X 891e5-6.

7 Idem, 892a 4-7. 
parle d'antériorité dans la naissance, il suggère ainsi une caractérisation de l'âme en général (que signale le singulier aủiñ s par rapport au pluriel $\sigma \omega \mu \alpha \dot{\tau} \tau \omega v)$ : on voit mal dans ces conditions comment attribuer à celle-ci une naissance qui ne vaudrait que pour certaines âmes.

On peut toutefois sortir de cette difficulté et réconcilier ce dernier texte avec le précédent (et avec Phèdre, 245d8), en considérant que l'âme naît ou advient à l'existence en tant qu'elle s'engage dans la génération d'autre chose ${ }^{8}$. Cela ne signifierait pas que, prise hors de son activité causale, elle soit proprement engendrée. Il faudrait tout simplement admettre que dès qu'elle entre dans un procès de production, elle précède naturellement ce qu'elle produit; en ce sens-là, il faut bien lui reconnaître une antériorité du point de vue de la genèse. Cela permet de comprendre les phrases étranges de l'Athénien, par exemple celle selon laquelle «ce qui est relatif à l'âme est advenu avant ce qui convient au corps"', signalant ainsi que l'âme acquiert un caractère premier relativement à quelque chose qui vient après, et pour autant qu'elle est prise dans un devenir dans lequel elle occupe nécessairement une position antécédente. Elle advient donc comme première dès lors que quelque chose naît comme second. Cela n'implique nullement qu'elle soit engendrée de façon absolue, par succession à un non-être. Ce qui naît, en quelque sorte, n'est pas l'âme elle-même, mais son caractère premier.

Évidemment, cette solution présuppose une distinction implicite entre la relation à soi de l'âme et sa relation à autre chose: c'est sous ce dernier aspect qu'elle naît comme antécédente, en lien avec une réalité autre qui doit être considérée comme postérieure (et c'est vrai de toutes les occurrences du verbe gignomai appliqué à l'âme dans les Lois). Dans les Lois, l'âme est toujours saisie dans son rapport à un autre. On vient de le voir à propos de la génération où le caractère engendré n'advient à l'âme que pour marquer son antériorité causale eu égard au corps qui naît ou qui est né. Mais on le constate aussi dès lors qu'il s'agit de définir le type de mouvement qui lui appartient en propre (ou, plutôt, de définir l'âme par ce type de mouvement). Dans la classification des

8 Un problème similaire se pose dans le Philèbe (27b) à propos de la cause et de la génération des trois autres genres. Voir F. Teisserenc, "D'une causalité démiurgique en cosmologie et en éthique», p. 345-346.

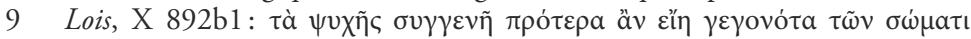

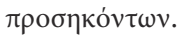


mouvements, c'est celui que l'Athénien et ses interlocuteurs s'accordent à reconnaitre comme premier: ils le caractérisent comme...

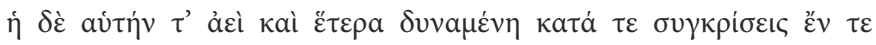

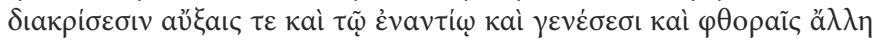

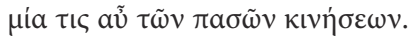

"Ce mouvement autre et unique parmi tous les autres mouvements, capable de se mouvoir toujours lui-même et les autres choses, que ce soit en des compositions ou en des décompositions, des augmentations et des diminutions, et des genèses et des corruptions ${ }^{10}$.

Cette insistance sur tout ce dont ce mouvement est cause atteste qu'il est essentiellement vu dans ce qu'il engendre en d'autres choses (car ce n'est pas à lui-même que s'appliquent la composition, la division, l'augmentation et la diminution, ou la genèse et la corruption). C'est encore ce qui est précisé dans les lignes suivantes où il est dit que ce mouvement s'harmonise (à titre de principe, pouvons-nous soupçonner) avec toutes les actions et toutes les passions ${ }^{11}$, qu'il peut donc être appelé le mouvement et le changement réel de tous les êtres ${ }^{12}$. On ne saurait mieux souligner la connexion forte du mouvement autoproduit avec tout ce qui est hors de lui, dont il est le principe de transformation.

De plus, l'Athénien rend explicite l'axiome (lequel était resté implicite dans le Phèdre) de l'impossibilité d'une régression à l'infini dans la série des mouvements transmis, et la nécessité de poser un premier moteur, le principe de cette série étant dans la chose qui se meut elle-même ${ }^{13}$. Cette chose peut d'autant mieux transmettre en dehors d'elle son propre mouvement qu'elle a d'entrée de jeu été posée comme ce qui se meut soi-même et ce qui meut autre chose, le kai grec (le «et" français) introduisant innocemment une conjonction pourtant problématique.

Dans ce passage, le terme "âme» est au singulier. Elle est, nous dit l'Athénien, source de mouvement. Mais comment entendre ce singulier? Faut-il y voir un terme collectif, qui désigne une classe d'êtres («les âmes»), un terme générique qui renvoie à un matériau ou un élément

10 Idem, 894b9-c1.

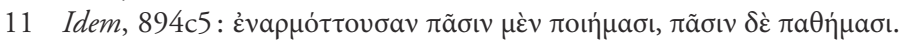

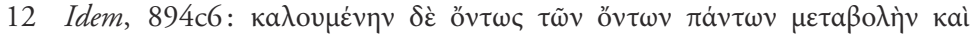
кívๆбı.

13 Idem, 894e5-895a3. 
(comme l'eau, la terre, le feu), ou bien est-ce le nom d'un principe unique et individué, qui communiquerait à l'ensemble des choses leur mouvement?

Examinons a contrario les exemples d'emploi du pluriel. L'Athénien situe les âmes à l'intérieur des corps, faits de terre, d'eau et de feu (895c5). Il y a de l'âme dans les corps vivants, et c'est cela qui les fait vivre, en les mettant en mouvement. Le ciel et les astres sont aussi animés par l'âme (car, à l'évidence, ils sont en mouvement) ${ }^{14}$. Là aussi, cette animation s'effectue de l'intérieur de ces corps, qu'ils soient terrestres ou

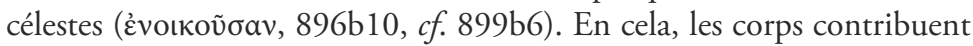
à individualiser l'âme qu'ils abritent et qui est leur moteur interne.

À propos de la genèse des êtres vivants, l'Athénien explique que l'âme présente dans un corps ponctuel le déploie progressivement dans les trois dimensions de l'espace (du point à la ligne, de la ligne à la surface, et de la surface au volume, 894a). Le corps acquiert alors une certaine figure (une hexis) et il demeure ce corps tant qu'il conserve cette hexis (le principe d'identité est fourni ici par la configuration spatiale du corps).

À ces exemples d'individuation et de multiplicité des âmes par les corps qu'elles animent ou qu'elles constituent, s'ajoute l'usage du pluriel dans le passage où sont distinguées les diverses espèces de mouvement autres que celui de l'âme proprement dite: il s'agit de choses qui se meuvent tantôt d'une manière, tantôt d'une autre, ou qui restent immobiles.

En revanche, dès lors qu'il est question de l'espèce du mouvement qui se meut lui-même et les autres, le texte adopte le singulier; appelé "le mouvement et le changement de tous les êtres ${ }^{15}$, il est également désigné comme "le principe de tous les mouvements, soit pour ce qui était immobile, soit pour ce qui est mû $»^{16}$.

Cette causalité universelle est d'interprétation délicate: est-elle celle de l'âme du monde, qui mettrait en mouvement l'ensemble de toutes les réalités incluses dans le monde? Or, si c'est là le seul principe, que deviennent les âmes particulières? Ne sont-elles pas, elles aussi, automotrices? Ne doivent-elles pas, à leur tour, être considérées comme

14 Idem, 898d4-5.

15 Idem, 894a5.

16 Idem, 895b4-5. 
des principes de mouvement? Impossible alors de parler d'un principe ou d'un mouvement au singulier...

$\mathrm{Si}$, en revanche, l'on décide d'entendre par «âme» ou "principe» telle âme ou tel principe de mouvement, c'est l'universalité de ses effets qui surprend: comment un principe local pourrait-il être la source de tous les mouvements dans l'univers?

Cette contradiction pourrait, à première vue, être levée en admettant qu'une âme originelle donne naissance à d'autres âmes, principes quand même de mouvement, mais subordonnés parce que seconds. Mais voilà qui entre en tension avec l'affirmation tout à fait générale que "l'âme" est première et toujours antérieure au corps. Comment est-ce possible si, de fait, certaines âmes sont dérivées?

Un double argument étrange vient ajouter à la confusion.

1. À supposer que le tout soit devenu immobile, d'où (re)naîtrait d'abord le mouvement? Réponse: de l'âme (895a5-b5).

2. À supposer que l'âme disparaisse, que se passerait-il ? Réponse: toute la genèse des vivants serait compromise (904b1). Dans le Phèdre, une question similaire était posée: à supposer que l'âme disparaisse, qu'en résulterait-il pour le tout? Réponse: dans son ensemble, il viendrait à s'immobiliser (245e1-2).

Dans tous les cas, il ne peut s'agir d'âmes individuelles, qu'elles soient celles d'animaux ou celle du ciel: aucune de ces âmes, en tant qu'elle est individuelle, ne peut, en disparaissant, immobiliser autre chose que son corps, et non la totalité des êtres présents dans le tout. Et aucune ne peut ranimer autre chose que son corps, et non la totalité des êtres présents dans le tout.

La seule façon de rendre intelligibles l'ensemble des arguments des passages cités des Lois (comme de celui du Phèdre), c'est d'admettre que le mot "âme», quand il est au singulier, ne renvoie pas à une âme, ni à un ensemble dénombrable d'entités psychiques, mais à l'élément psychique, à un matériau répandu et dispersé dans l'univers (il renvoie donc à "de l'âme»). Il en va de même avec les termes "principe» et même "mouvement»: ils nomment ce matériau dans son genre, mais sans diviser ses parties ${ }^{17}$. Le raisonnement à cet égard est comparable à

17 Sur la grammaire et l'ontologie implicite de ces termes «qui ne divisent pas leur référence», voir W. Quine: «les termes dits de masse comme "de 
celui d'un cosmologiste contemporain qui soutiendrait que sans eau, il n'y a pas de vie possible.

Du reste, tant la présentation de la doctrine des athées que son inversion platonicienne traitent de manière équivalente, sur un plan sémantique, les éléments et l'âme (891c, 892c). Le différend porte sur la priorité qu'il faut accorder aux uns par rapport à l'autre. Sont mis en balance, d'un côté, l'eau, le feu ou la terre, et de l'autre, l'âme. Or, pas plus que lorsque l'on évoque la terre, n'est-ce une poignée de terre singulière qui est désignée, pas plus, lorsqu'il est question de l'âme, n'est-ce une âme singulière qui est visée. La difficulté est que nous ne sommes guère habitués à traiter le terme "âme" comme un mass term (un «nom de masse»). Sans doute du fait de la religion chrétienne, nous avons tendance à prendre l'âme comme synonyme de personne, comme un nom qui nomme soit un individu, soit un groupe d'individus, et non une sorte de matériau. Il faudrait réussir à entendre, quand ce terme est au singulier, non une âme individuelle, mais "ce qui est âme».

En passant, il en va de même du terme "corps»: lui aussi est entendu comme un mass term. Sinon la proposition platonicienne selon laquelle "l'âme est plus ancienne que le corps" serait tout à fait étrange: comment l'âme d'un nouveau-né serait-elle plus ancienne que le corps d'un vieillard? Il ne peut s'agir d'âme individualisée par son incorporation, ni de corps dont l'identité soit définie par sa figure. Mais l'antériorité de l'âme sur le corps est comparable à l'antériorité de la vapeur d'eau sur la pluie: pas de pluie sans vapeur d'eau préalable, ce qui ne veut pas dire que "cette pluie-ci» est nécessairement postérieure à "cette vapeur d'eau là".

Et dans le passage des Lois qui évoquait les conséquences désastreuses résultant de l'anéantissement hypothétique de l'âme, le corps est considéré à l'égal de l'âme:

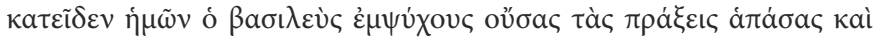

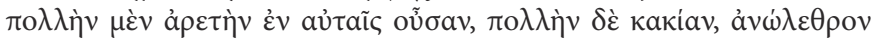

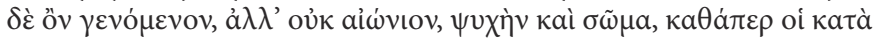

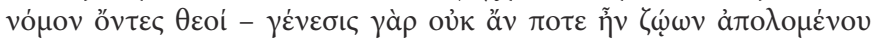

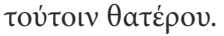

l'eau"... ont la propriété de référer cumulativement: toute somme de parties qui sont de l'eau est de l'eau" (Le Mot et la Chose, p. 141-142). 
"Notre roi s'aperçut que toutes les actions émanent de l'âme, et qu'en elles il y a beaucoup de vertus comme beaucoup de vices, l'âme et le corps étant nés indestructibles, quoique non éternels, exactement comme le sont les dieux selon la loi - car il ne pourrait y avoir de naissance d'êtres vivants si l'un ou l'autre venait à périr " ${ }^{18}$.

Ce passage soutient la nature indestructible de l'âme et du corps, et souligne l'impossibilité d'une genèse des êtres vivants si l'un des deux matériaux venait à manquer. Âme et corps sont vus comme des principes non individués (à coup sûr, l'âme du monde n'est pas concernée ici, en tant que telle: comment serait-elle susceptible d'actions viciées, alors qu'elle est pilotée par l'intellect?) Ce sont des matériaux impérissables, qui peuvent être, dans la suite, diversement découpés et ajustés l'un à l'autre pour former chacun des êtres vivants, mais qui sont ici considérés de façon absolument générique, la référence aux dieux selon la loi (tant les dieux dont Platon décrit par un muthos la fabrication dans le Timée que les dieux olympiens, immortels tout en étant nés) introduisant une comparaison avec les seules réalités individuelles dont le sort est comparable à l'âme et au corps pris comme élément et étoffe du monde.

D'ailleurs, l'Athénien exclut l'éventualité qu'une âme en sa singularité vienne à se former indépendamment du processus de recomposition du matériau psychique. Quelque chose qui serait originellement dépourvu de la propriété de l'automotricité ne peut en être postérieurement doté. Cela supposerait, en effet, qu'un corps $\mathrm{x}$ reçoive du dehors un mouvement et que ce mouvement communiqué lui communique aussi la faculté de se mouvoir lui-même. Or l'Athénien caractérise ainsi le mouvement reçu d'un autre en un autre (que celui qui l'a donné):

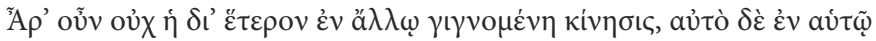

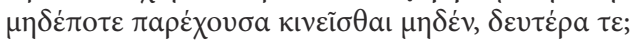

"N'est-ce donc pas que jamais ce mouvement produit par un autre en un autre ne dispensera à quoi que ce soit le pouvoir de se mouvoir soi-même en soi-même, et qu'aussi il est toujours second?»" ${ }^{19}$

Selon l'Athénien, il est donc impossible qu'une âme transmette à quelque chose d'autre (qu'elle met en mouvement) le pouvoir de se mouvoir soi-même.

19 Idem, 896b5. 
Une conclusion s'impose: le stock d'âme est défini une fois pour toutes ${ }^{20}$. Une quantité de substance psychique invariable circule dans l'univers, où elle se trouve découpée selon les types de corps en lesquels elle se distribue, exactement comme la vapeur d'eau, qui est cause universelle de la pluie, se trouve concentrée tantôt ici, tantôt là.

Autre conséquence de l'âme entendue comme une sorte de matériau continu et diffus: son individuation par le corps est, dans une certaine mesure, extérieure et précaire. D'où la facilité avec laquelle Platon adopte le thème de la palingénésie: l'âme se répand dans les choses, passe d'un vase à un autre ${ }^{21}$, l'anime puis le quitte pour en mouvoir un troisième et ainsi de suite.

\section{L'irrationalité de l'âme nue}

Le mouvement psychique, dont on dit qu'il est premier dans l'ordre de la génération, n'est jamais caractérisé comme circulaire, pas plus dans les Lois que dans le Phèdre $e^{22}$. Ce n'est pas non plus le cas du mouvement second dont il est la cause prochaine.

Ce point est-il significatif? Oui, car il indique que la circularité est une donnée contingente pour l'âme, mais caractéristique pour sa part de l'intellect. Elle marque l'étape intermédiaire entre une approche physique ou cosmologique, attachée à identifier une source du mouvement au sens strict, et une approche psychologique, attachée à dévoiler les sources cognitives et intentionnelles du comportement.

A contrario, si l'on regarde les définitions du mouvement de l'intellect dans les Lois, bien qu'elles soient formulées dans un langage physique puisqu'il est question de kinèsis, de lieu, de distance, d'égalité de rapports, rien n'indique en elles la mention d'un mouvement automoteur ${ }^{23}$.

20 Cf. République, X 611 a.

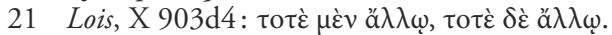

22 Contrairement à ce que soutiennent L. Brisson et J.-F. Pradeau (Platon,

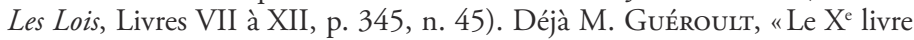
des Lois et la dernière physique platonicienne», p. 43: "L'âme principe du mouvement comme mouvement spontané et se suffisant à soi, n’est adéquatement représentée que par un mouvement circulaire [890a]». Ni ce renvoi, ni aucun texte dans les Lois ne justifie cette affirmation.

23 À la différence de Timée, 37b5. 
Enfin, le fait que l'âme première née est automotrice et se définit exclusivement ainsi, ouvre en droit la possibilité qu'elle revête les caractéristiques inverses de celles de l'intellect. S'il avait été en effet accordé à ce mouvement automoteur une allure circulaire, il eût été difficile de refuser à l'âme une dimension intelligente. Et toute âme, en tant qu'elle meut et se meut, aurait dû alors être caractérisée par son intelligence. Or c'est là un résultat dont ne veut manifestement pas l'Athénien: en effet, il faudrait admettre que les animaux, s'ils sont proprement doués d'âme, sont aussi doués d'intellect, contrairement au privilège accordé à l'âme humaine et aux dieux selon le Phèdre. L'Athénien évoque clairement la possibilité d'une âme dépourvue de toutes les caractéristiques de l'intellect, dépourvue de phronèsis et d'arete ${ }^{24}$.

Une objection vient naturellement à l'esprit: l'Athénien ne cherchet-il pas à établir l'antériorité, par rapport au corps, de l'âme et de ce qui lui est "apparenté»? Pour expliquer ce qu'il entend par cette parenté, il fait état, dans une première énumération, de «l'opinion, le soin, l'intellect, la technique et la loi» (892b). C'est un ensemble assez composite, que caractérisent d'une part une dimension cognitive, d'autre part une orientation vers un but. Ce dernier, pour au moins quatre des éléments énoncés, est un but pratique, en cela que l'activité psychique vise à disposer d'une certaine manière des corps. Dans une seconde énumération, sont mentionnés les «tempéraments, les mœurs, les souhaits, les raisonnements, les opinions vraies, les soins, et les souvenirs" (896c-d). Cette dernière liste appelle les mêmes remarques que précédemment, renforcées pour ainsi dire par l'absence remarquable de l'intellect. Quelle parenté établir alors entre cet ensemble de phénomènes et l'essence cinétique de l'âme?

Avant même d'en saisir la nature, le lecteur en comprend la nécessité dans le cadre d'une lutte contre l'athéisme: s'il faut montrer que c'est l'art qui est premier dans la genèse des choses (et non le hasard ou

24 Lois, X 896e5-6, 897b9. Un certain nombre de commentateurs associent l'âme et l'intellect; or, quoi qu'en dise S. Menn par exemple (Plato's God as Nous, ch. 4), s'il n'y a pas d'intellect sans âme (Philèbe, 30c; Timée, 30b; Sophiste, 248d-249d), il y a des âmes sans intellect. "Reason (nous) inheres in soul, and is present where soul is" (J.Abolafi, "Solar Theology and Civil Religion in Plato's Laws", p. 379): seule la première partie de la phrase est vraie. 
une nature aveugle), il convient alors de lier la psukhè, dont la priorité cinétique vient d'être élucidée, à tout ce qui est activité téléologique ou intelligente, afin que cette dernière jouisse de la même priorité. Toutefois, on bute sur un écueil déjà présent dans le Phèdre. La redéfinition de l'âme par son caractère automoteur, définition qui a un sens d'abord physique, ne permet pas en elle-même de lui rattacher tout ce qui relève de la psychologie ordinaire.

Cela est d'autant plus remarquable qu'une brève réflexion (895d-896a) sur le rapport entre nom / logos / chose, une des plus nettes dans le corpus platonicien ${ }^{25}$, précède la définition de l'âme. L'Athénien et Clinias disposent d'une définition et sont à la recherche d'un nom pour la chose définie. Le nom se trouvant affecté à la définition doit recevoir exclusivement son sens de celle-ci.

Or le coup de force platonicien, en identifiant l'«âme» avec ce qui se meut soi-même, consiste à toujours inclure dans le nom, et donc dans la chose nommée, des aspects de son sens ordinaire qui ne sont pas logiquement dépendants de la définition rigoureuse qui vient de lui être allouée.

"L'Athénien. - Ainsi, tu affirmes que "se mouvoir soi-même" est la définition qui appartient à cette même réalité que pour ce qui est du nom, nous appelons tous "âme"?

Curnias. - Oui, je l'affirme ${ }^{26}$.

Le diable ici se cache dans le "tous»; il va précisément permettre de tirer la conclusion qui intéresse l'Athénien.

«L'Athénien. - Nous nous rappelons être tombés d'accord précédemment que si l'âme se révélait à nous être plus ancienne que le corps, également ce qui appartient à l'âme serait plus ancien que ce qui appartient au corps?

Clinias. - Hé, oui absolument.

L'Athénien. - Le tempérament, le caractère, les volitions, les raisonnements, les opinions vraies, les soins ainsi que les souvenirs doivent donc être nés antérieurement à la longueur, à la largeur... . ${ }^{27}$

25 Voir aussi Sophiste, 221b1, Théétète, 201e-201c, Lettres, VII 342b.

26 Lois, X 896a4-5

27 Idem, 896c5-d2. 
Cet accord préalable concédé plus haut (892b) pouvait apparaitre à ce moment-là légitime, car l'âme n'avait pas encore reçu sa définition stricte de réalité automotrice. Il ne l'est plus maintenant, sauf à démontrer l'unité de ces deux familles, les mouvements automoteurs d'une part, les diverses activités cognitives et conatives d'autre part.

Une difficulté toute particulière tient aux activités cognitives: autant l'idée que les désirs meuvent le corps est une idée familière et intuitivement recevable (encore qu'on ne perçoive pas directement la nature cinétique du désir, comme nous l'expliquerons dans un instant), autant l'idée d'une activité cognitive qui meut un objet ${ }^{28}$ apparaît contreintuitive: le savoir ni ne déplace ni ne transforme son objet, et c'est plutôt l'âme qui connaît qui pâtit de l'objet connu (et donc, si l'on veut, est mue par ce dernier ${ }^{29}$ ).

Il est en outre probablement impossible à l'intellect de mouvoir quoi que ce soit - il est mû sans mouvoir quelque chose d'autre, sauf, peutêtre, à la manière du monde dans son ensemble, c'est-à-dire d'une sphère qui meut tout ce qui est en elle (contrairement à l'action standard de l'âme qui meut en étant dans la chose mue). En effet, ce mouvement de l'intellect est conçu essentiellement sous le signe du même. Il se définit par l'identité de rapport, de distance, de direction et de lieu. Il est donc privé de tout lien à une altérité susceptible d'être mue.

Mais le domaine des désirs au sens large (aversion, amour, opinion droite qui touche au préférable, souhaitf n'est pas non plus sans poser de graves problèmes du point de vue même de la doctrine du mouvement qu'élabore ici l'Athénien. Sans doute, l'assimilation du désir à un mouvement de l'âme découle-t-elle assez naturellement de deux présupposés largement répandus - que l'Athénien partage -, l'un qui admet l'intériorité de l'âme au corps, l'autre qui reconnaît une causalité au désir: qui nierait en effet que la cause du fait qu'un homme se lève et se serve un verre d'eau est qu'il a soif? Puisque le désir de boire est à l'origine du mouvement visible du corps, l'incitation est grande à faire du désir lui-même un mouvement qui cause un mouvement (car

28 Rappelons que le mouvement premier, auquel est identifiée l'âme, est un mouvement qui se meut lui-même et qui meut autre chose (894a9-10).

29 Voir Sophiste, 248a-249d. Sur le sens exact de ce dernier texte, voir F. Teisserenc, "Puissance, activité et passivité dans le Sophiste», p. 25-45. 
comment ce qui est au repos pourrait-il produire un mouvement?). Voilà l'intuition sur laquelle s'appuie ici l'Athénien.

Toutefois, la typologie des mouvements qu'il vient de développer souligne leur dimension spatiale: tous se déroulent soit dans un lieu, soit dans plusieurs lieux (893c, réitéré de façon solennelle en 898a). D’où la question: si le désir est un mouvement, quel(s) est (sont) le (les) lieu(x) du désir?

De plus, l'Athénien propose une distinction entre mouvement linéaire et rotation. Le désir sera-t-il alors linéaire ou circulaire? Quelle sera sa vitesse? Comment se divisera-t-il et comment se composera-til? Le même Athénien évoque des mouvements de direction opposée. Des désirs contraires seront-ils également des mouvements de direction opposée? Mais comment dire que le mouvement du désir va vers le haut ou vers le bas? Vers la droite ou vers la gauche?

Enfin, lier le désir à l'automotricité de l'âme implique, semble-t-il, que le désir est produit par l'âme seule, d'elle-même en elle-même. Or comment la faim, par exemple, pourrait-elle être auto-engendrée dans l'âme? Comment l'âme pourrait-elle, en elle-même et d'elle-même, susciter de la douleur, sans faire intervenir un pâtir impliquant une cause extérieure?

Que Platon soit conscient d'une grave difficulté en ces différents points se manifeste justement par la référence à deux âmes, l'une rationnelle, l'autre irrationnelle. Cette dualité, sur laquelle on reviendra, exprime non seulement une distinction entre le noétique et ce qui ne l'est pas, mais encore une tension entre l'approche physique et l'approche "psychologique".

L'Athénien est très clair: il signale la contrariété des effets physiques (croissance, composition, génération d'un côté, décroissance, division, corruption de l'autre) qui sont rapportés à la causalité de l'âme, qui tient à ce qu'elle est précisément la source universelle de tous les mouvements et de tous les changements ${ }^{30}$.

Mais il montre également comment les mouvements premiers, ceux que l'âme se procure à elle-même et au corps, sont divisés quant à leur orientation cognitive et éthique. L'Athénien mentionne l'opinion vraie comme l'opinion fausse, ainsi que tous ces mouvements contraires que

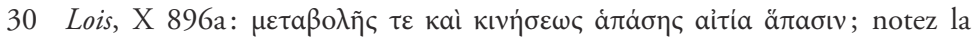
répétition de l'adjectif ärac. 
sont l'amour et la haine, le plaisir et la peine, la confiance et la crainte (897a). L'âme est dès lors la cause du bien et du mal, du beau et du honteux, du juste et de l'injuste et de tous ces contraires (896d5).

À l'évidence, de telles propositions ne sont guère compatibles avec celle de l'antériorité de l'art sur la nature, découlant de l'antériorité de l'âme sur le corps. Car le désordre et l'absence de mesure produits l'un et l'autre par l'âme (906b) peuvent difficilement passer pour une tekhnè.

\section{L'automotricité de l'âme et l'existence divine}

D'où la perplexité du lecteur: comment comprendre que la démonstration de l'existence des dieux (et de leur providence) soit amorcée par une réflexion sur l'âme automotrice? Car cette thèse n'est pas nécessaire au résultat que vise l'Athénien, dans la mesure même où la priorité physique de l'âme quant au mouvement n'implique nullement la priorité ordonnatrice de l'intellect et de ce qui en émane, l'art, la loi, les constructions intelligentes.

Ajoutons même que la thèse de l'automotricité est contreproductive, puisqu'elle reconnaît la présence d'une substance psychique en tous les vivants, leur accorde de ce fait la propriété de spontanéité - de commencer absolument une série causale - et se prive, par la même occasion, de la possibilité de remonter à un premier moteur unique et remarquable. La conséquence indésirable de cette thèse, du point de vue d'une démonstration de l'existence divine, c'est la banalisation des premiers moteurs, qui pullulent dans toute la nature et ne sont nullement réservés aux astres.

Suffirait en revanche à justifier l'existence des dieux le caractère circulaire du mouvement cosmique, auquel est analogue le mouvement de l'intellect. Au vu des révolutions régulières des astres, et notamment du soleil, l'idée doit s'imposer que ces derniers sont pilotés par des intelligences ou des âmes intelligentes. Ce qui ne veut pas dire que l'intellect soit, en tant que tel, automoteur; est seulement requis que le facteur qui produit et règle le mouvement des astres (qu'il l'ait reçu ou qu'il l'engendre de lui-même) soit doté d'intelligence.

L'argument, toutefois, n'est opérant qu'à condition que seule l'âme soit réellement cause des mouvements, et qu'elle les cause de deux façons seulement, en produisant des mouvements réglés ou ordonnés ou en produisant des mouvements déréglés. L'Athénien précise bien que ces 
deux modes opératoires de l'âme épuisent exhaustivement l'ensemble des phénomènes concernés:

i) quand l'âme s'associe à l'intellect (voũc), elle guide toutes les choses qui ont atteint leur rectitude et leur bonheur;

ii) quand elle est liée à l'ignorance insensée (ävoı $\alpha$ ), ce sont toutes les choses contraires dont elle est la cause (897b1-5). Il est donc possible d'imputer toutes les irrégularités à la seule âme irrationnelle, et à l'inverse, l'âme noétique est la seule origine de l'ordre et de la régularité des entités considérées.

L'ensemble équivaut ainsi à reconnaître deux axiomes préalables. D'abord, un axiome d'unicité causale, en vertu duquel existe une bijection stricte entre l'âme noétique et la production première d'un mouvement ordonné, et entre l'âme irrationnelle et celle d'un mouvement désordonné. Grâce à cette double bijection, il serait possible de remonter de l'effet observé à sa cause première invisible, et de dire si, oui ou non, le mouvement du monde est imputable à un agent rationnel. Il faut toutefois ajouter un autre axiome, de conservation ou de transitivité, implicite dans les propos de l'Athénien, pour que l'opération d'imputation soit valide. Il convient en effet que la transmission du mouvement ne modifie pas la qualité du mouvement transmis. L'aspect ordonné du premier d'entre eux doit se répercuter tout le long de la chaîne des mouvements, comme, à l'inverse, doit se maintenir son éventuel aspect désordonné. Il n'y a pas de mouvement ordonné qui dégénère, ou de mouvement désordonné qui se rectifie, sinon par intervention d'un mouvement tiers, lui-même désordonné ou à l'inverse ordonné. Dès lors, à partir de la présence d'un désordre, on peut en toute sécurité remonter à une source irrationnelle, et partir de celle de l'ordre, à une source rationnelle. Car deux mouvements désordonnés qui se combinent ne peuvent pas donner naissance à un mouvement ordonné, ni, inversement, deux mouvements ordonnés ne peuvent faire surgir un désordre.

Cet axiome permet d'écarter la suggestion des athées: selon ces derniers, on s'en souvient, l'ordre (les saisons, le ciel et ce qu'il contient, les vivants) surgit du mélange des contraires (le chaud et le froid, le sec et l'humide) et naît du hasard des rencontres et des combinaisons qui se réalisent selon la "puissance" des termes qui se "tombent" dessus (889b6). De telles mutations sont exclues par la règle de transitivité et de conservation. C'est là une position de principe, en faveur de laquelle l'Athénien ne fournit pas directement d'arguments. Le lecteur peut 
regretter cette carence, qui fragilise considérablement la tentative de démonstration de l'existence des dieux, et, surtout, ne permet pas de réfuter proprement les athées, mais seulement de s'opposer à eux.

Toutefois, cet axiome de transitivité ou de conservation, combiné à celui de causalité psychique première et unique, permet d'éviter un grossier non sequitur. En effet, si l'âme n'était pas cause universelle, si sa causalité ne comportait pas une double modalité exclusive de toute autre causalité pour ce qui concerne l'apparition du caractère régulier/ irrégulier au sein de la succession des mouvements, alors la présence d'un mouvement circulaire ne prouverait rien. À elle seule, l'existence d'un rapport orienté de causalité entre intellect et circularité, à supposer qu'elle soit accordée, n'implique nullement le rapport converse. Un

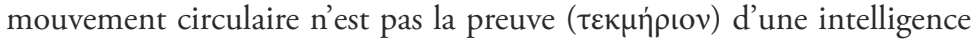
à l'œuvre: "si a alors b" (si une intelligence dans une âme, alors un mouvement circulaire produit par celle-ci), ne veut pas dire «si b alors a" (si un mouvement circulaire, alors une intelligence qui le cause). Cette erreur n'est donc évitée que par la règle «les mouvements réguliers sont tous produits par l'âme rationnelle et les mouvements irréguliers par l'âme irrationnelle», combinée à un principe de non-émergence («en cas de mouvements reçus et communiqués, l'ordre ou le désordre passe des uns aux autres et ne fait pas l'objet d'une genèse de novo").

Reste que l'alternative claire et efficace entre le régulier (l'ordre) et l'irrégulier (le désordre), indispensable à la conclusion du raisonnement en faveur de l'existence des dieux, se trouve en partie brouillée par l'introduction implicite d'un troisième principe, le principe de classement par proximité d'avec le pôle de perfection que représente le mouvement de l'intellect pur.

À la révolution de l'intellect, suggère l'Athénien, seront semblables

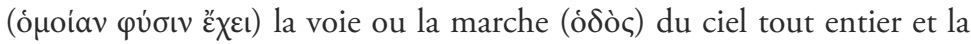
translation ( $\varphi$ o ò) de tous les êtres qu'il contient (897c). Pour mesurer cette similitude ou cet écart, il faut à tout le moins disposer d'une «image» de ce mouvement de l'intellect. Cette image est fournie par la rotation du cercle, mais aussi par celle de la sphère, rotation qui doit en outre satisfaire les conditions suivantes, comme meilleure approximation :

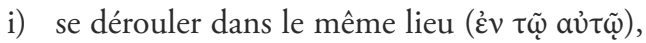

ii) avec une vitesse constante ( $\dot{\omega} \sigma a v ́ \tau \omega \varsigma)$,

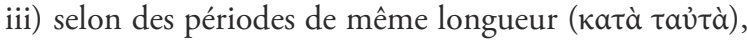

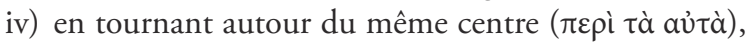




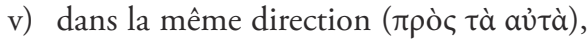

vi) selon une formule déterminée ( $\kappa a \theta^{\prime}$ '̌v $v a \lambda$ ó $\left.\gamma o v\right)$ et

vii) en gardant un ordre unique ( $\tau \dot{\alpha} \xi ı \nu \mu i \alpha v)$.

Il résulte de la caractérisation de ce mouvement qu'i i est théoriquement possible de lui opposer des mouvements

i) qui se déroulent en plusieurs lieux (et non en un seul mouvements circulaires et linéaires donc, au lieu de la simple rotation),

ii) qui se produisent avec des vitesses changeantes (et non pas constantes),

iii) selon des périodes irrégulières (et non pas régulières) d'accélération et de décélération,

iv) autour d'un centre changeant (épicycle ou tout autre mouvement autour d'un point lui-même mobile),

v) en procédant à un renversement de direction (comme le monde dans le mythe du Politique),

vi) selon des durées de longueur changeante (au lieu d'un renversement périodique),

vii) au sein desquelles l'accélération et la décélération elles-mêmes peuvent se modifier de manière aléatoire ${ }^{31}$.

Il en découle également que nous n'avons plus une simple alternative, comme tout à l'heure, entre le régulier et l'irrégulier. La négation cumulée de tous ces critères donne certes le modèle inverse, le paradigme du mouvement irrationnel. Mais entre le pôle de perfection et celui de l'imperfection, s'échelonnent les négations partielles, concernant un ou plusieurs critères. Plus on introduit de variations possibles et plus on s'éloigne de la simplicité du mouvement de l'intellect.

À partir de cette grille d'évaluation, l'assimilation de la révolution du ciel à un mouvement parfaitement uniforme sur tous les plans permet de conclure à sa direction par l'âme la plus excellente (dotée de l'intellect le plus efficace), donc à la présence d'un ou plusieurs dieux dans l'univers, la rotation des astres les plus visibles (le soleil, la lune) impliquant la présence d'une âme singulière qui les pilote, soit directement de

31 La nature elliptique de la notation platonicienne n'autorise ici que des conjectures sur la nature des identités et des changements envisagés. 
l'intérieur, soit par le truchement d'un corps intermédiaire, soit encore d'une autre façon qui reste inexpliquée ${ }^{32}$.

Ce détour par le mouvement de l'intelligence, ou de son image, n'a donc pas été inutile pour le raisonnement d'ensemble de l'Athénien. Il a permis, en hiérarchisant les mouvements, d'apprécier l'excellence de leur cause: pas seulement d'identifier une source intelligente à l'origine du mouvement de l'univers, mais encore une source superlativement intelligente - divine ${ }^{33}$.

Ajoutons, toutefois, que de la structure même de l'argument, il découle que quasiment n'importe quel mouvement attribué à l'univers aurait permis de conclure, selon la même fallace que dans le cas du mouvement rotatif, à l'intelligence relative de sa cause, car il aurait présenté, à un titre ou un autre, une forme de régularité. Sauf à considérer le cas où le mouvement observé aurait donné lieu à une négation de tous les critères d'identité, la présence d'un ou de quelquesuns d'entre eux devrait pouvoir suffire à reconnaitre la présence d'une âme intelligente. Mais intelligente jusqu’à quel degré et selon quelle puissance? Difficile à préciser.

Demeure le parallèle suivant: de même que la réflexion sur la cause automotrice avait dû aboutir à un panpsychisme, de même la réflexion sur la régularité du mouvement débouche sur une pannoétique. La frontière entre le divin et le non-divin s'estompe, elle laisse la place à une gradation continue d'un extrême à l'autre. Dès lors qu'on est amené, d'une façon éminemment discutable, on l'a vu, à conclure à l'existence d'un dieu intelligent à partir de la révolution d'un astre, on est également amené, en prenant en considération la plupart des mouvements de l'univers, à conclure que "tout est plein de dieux»" ${ }^{34}$, comme le dit l'Athénien reprenant une formule de Thalès et d'Héraclite.

Faisons un bilan de cette "preuve».

32 Lois, X 898e5-899a5.

33 Largument platonicien est donc distinct des arguments de théologie naturelle qui reposent sur l'idée d'un "design» intelligent. Le monde ici n'est pas façonné par Dieu, ce n'est pas un artefact dont la sophistication obligerait à mettre à son origine une cause démiurgique. En revanche, selon l'Athénien, certaines parties du monde expriment, pour ainsi dire, la conduite d'un dieu. Voir N. Powers, "Plato's Cure of Impiety in Laws X", p. 55-56.

34 Lois, X 899b9. 
1. Elle débute par la considération de l'automotricité. De la nouvelle définition de l'âme qui en est issue ne se laisse pas déduire l'inclusion des activités cognitives et conatives dans une cinétique générale, contrairement aux affirmations de l'Athénien. En outre, le thème de l'automotricité ne semble guère contribuer à la démonstration des dieux, puisque cette automotricité peut aussi bien être rationnelle qu'irrationnelle, source d'ordre comme de désordre.

2. Toutefois, elle a l'avantage de circonscrire l'enquête sur les causes du mouvement: toutes les familles de mouvement prennent leur point de départ dans une source unique, l'âme. La dualité des modes de production du mouvement par l'âme paraît devoir autoriser de remonter des effets aux causes, de conclure du mouvement ordonné de l'univers à une âme intelligente qui le produit.

3. Une telle remontée cependant n'est légitime que sur la base de deux axiomes ou de deux prémisses supplémentaires, en euxmêmes arbitraires: l'axiome de transitivité et de conservation des propriétés du mouvement, et celui de l'unicité causale de l'âme (de l'âme intelligente quant aux mouvements ordonnés, de l'âme irrationnelle quant aux mouvements désordonnés).

Du point de vue de son caractère démonstratif, la preuve est donc un échec. Faut-il pour autant en conclure que l'Étranger d'Athènes est aveugle aux failles de son discours, qu'il est dupe de sa propre rhétorique? Pas nécessairement. Il donne au contraire des indices de sa lucidité et paraît reconnaitre qu'il n'est pas en mesure de convaincre les incrédules ${ }^{35}$. Son ambition serait somme toute plus modeste: persuader les âmes bien nées ${ }^{36}$, en offrant du monde une image vraisemblable, qui n'est pas outrancièrement contredite par les phénomènes observables.

Au début de cette digression destinée à "prouver" l'existence des dieux, on se souvient peut-être que l'Athénien s'était mis en colère contre les impies, lesquels n'ont été persuadés ni par les mythes contés

35 N'envisage-t-il pas la peine de mort pour les athées honnêtes, que cinq ans d'entretiens clandestins avec les membres du conseil nocturne n'auraient pas suffi à convaincre (Lois, X 909a8)?

36 Pour une analyse fine du public auquel l'Athénien destine son propos, voir S. BRILL, "Psychologie and Legislation in Plato's Laws», p. 221-224. 
par les nourrices, ni par le spectacle (tragique) qui les accompagnait, ni par les rites qu'accomplissaient leurs parents et qu'ils adressaient à des dieux conçus comme des êtres absolument véritables (887e). L'Athénien accusait les impies de remettre en cause tout cela «sans raison suffisante", ainsi que les accuseraient ceux qui sont "pourvus d'un tout petit peu d'intelligence». Il dénonçait leur «pensée corrompue», qui le force, lui, à donner ses raisons, à faire preuve de voũ $\varsigma^{37}$. Il soupçonnait qu'à partir du moment où ces choses (les dieux et leur conduite) en viennent à être discutées, l'intelligence est en mal d'établir leur vérité, et donc que, comme intelligence $d u$ sujet, elle n'est pas tout à fait à la hauteur de l'intelligence $d u$ monde. Par sa nostalgie des temps anciens où les âmes accordaient en toute simplicité leur foi à l'enseignement religieux traditionnel, l'Athénien avouait à demi-mot qu'il serait plus à son aise si l'on pouvait confier au mythe la représentation d'un monde rationnellement organisé. L'intelligence ne peut pas fonder son propre fondement, devinait-il.

Après l'examen des tekmèria avancés par l'Athénien, nous constatons qu'il ne s'est pas trompé. Il n'a rien démontré en effet et n'a produit qu'un argument vraisemblable, bien digne d'être gravé sur la pierre pour l'édification des citoyens de Magnésie ${ }^{38}$.

Soit, dira-t-on. La valeur épistémique du discours de l'Athénien s'en trouve limitée, au profit de son usage politique et éducatif. Encore faut-

37 R. MaYhew («Persuasion and Compulsion in Plato’s Laws 10», p. 91-111) commente ce passage et souligne à juste titre que cette "haine» à l'égard de l'athée montre que chez l'Athénien (et chez Platon), la recherche de la persuasion rationnelle n'est pas un objectif désirable en lui-même, car ce qui est désirable est au contraire d'avoir des citoyens si profondément convaincus par les mythes qu'ils dispensent de cette persuasion (contre l'interprétation lénifiante de C. BовоNICH dans «Persuasion, Compulsion, and Freedom in Plato's Laws», p. 389). L'Athénien doit ainsi se livrer à une "démonstration" vue ici comme un pis-aller. Toutefois, R. Mayhew n'explique pas vraiment la «haine» que fait surgir ici l'athée dans le cœur non seulement des bons citoyens, mais aussi du législateur intelligent qu'est l'Athénien. La raison en est la crainte de l'Athénien qu'étant forcé par l'athée à faire paraître le voṽ ̧ qui doit établir la royauté cosmique du voṽc, il ne fasse aussi paraître sa singulière faiblesse.

38 L'ensemble du passage cosmologique des Lois, de façon exceptionnelle dans l'œuvre de Platon, écarte les deux autres personnages du dialogue en sus de l'Athénien et il a vocation à être couché par écrit, comme le sont les autres lois, pour l'instruction de tous les futurs citoyens. 
il que cette clarification des finalités et du statut de son propos aide à résoudre un certain nombre de problèmes toujours en suspens.

D'abord, reste sans réponse la question de l'intérêt de la thèse de l'âme automotrice, alors qu'elle ne constitue pas une prémisse indispensable pour le raisonnement en faveur des dieux. Si elle a permis d'établir une cause unique du mouvement, c'est, on l'a vu, selon deux modalités contraires. Il eût donc été parfaitement possible de réserver à la seule âme les mouvements réglés, et d'imputer les mouvements déréglés à une cause non psychique, à une "causalité errante», comme c'est le cas dans le Timée. Le résultat eût été le même. Pourquoi donc mettre autant en avant une idée dont Platon aurait pu faire l'économie dans le cadre de sa "preuve»?

Une autre question demeure ouverte: d'où vient que l'intelligence ait besoin d'un appui cosmologique pour déployer sa puissance à l'échelle humaine? Sans doute, une trop grande irrégularité du milieu paralyseraitelle les hommes. Mais il semble que, pour Platon, la subordination de leur intelligence à celle, putative, des dieux, soit une nécessité pratique et politique. En quel sens exactement, et comment?

\section{L'autonomie seconde de l'être humain}

Le thème de l'âme automotrice, on l'oublie trop souvent, a l'avantage considérable de souligner la continuité des hommes aux dieux, puisqu'ils partagent à cet égard une commune nature. En vertu de cette parenté, ce que font les uns est susceptible d'inspirer ce que les autres ont à faire. S'il y avait solution de continuité entre hommes et dieux, ceux-ci ne pourraient jamais constituer des modèles pour ceux-là.

Mais cette continuité de nature est aussi celle du réfléchi : les hommes comme les dieux sont à titre égal des êtres qui se meuvent. Voilà qui a comme conséquence que les uns comme les autres sont mutuellement indépendants. Les hommes ne doivent pas leurs mouvements aux dieux, et inversement. Ils sont cause première de leurs mouvements, comme les dieux le sont des leurs.

Cette indépendance est de principe. Elle constitue la forme négative de la liberté des hommes. Il se peut assurément que des facteurs externes bornent l'étendue et la variété des mouvements que les vivants sont susceptibles de produire. Mais cette limitation ne s'opère qu'à l'intérieur 
d'une gamme d'actions et de passions que fixe la nature de chaque être animé $^{39}$ (l'âme ainsi que le corps auquel elle s'est liée).

Un chien et une étoile n'ont pas le même pâtir et le même agir. Si chacun des deux se trouve intérieurement déterminé à produire selon sa puissance un certain type de mouvement, le premier plus que la seconde peut être empêché de le faire, du fait de son environnement et d'une certaine illimitation constitutive ${ }^{40}$. Pour ce qui est de l'homme, sa nature, comme celle de tous les vivants, engage un certain type de conduite -que ce soit en réaction à un pâtir déterminé ou en vertu de son propre dynamisme. Si des facteurs externes viennent à la modifier, c'est pour des raisons similaires à celles des autres animaux, mais c'est également parce que se glisse une forme de mimétisme dans certaines des opérations par lesquelles il produit son mouvement ${ }^{41}$. Ce rapport d'imitation est à mettre au compte de l'agent imitant, qui prend pour ainsi dire l'initiative (plus ou moins explicite) de faire d'un être situé hors de lui-même le modèle de sa conduite. L'agent reste ainsi l'auteur de son action, tout en laissant à un autre le soin de l'orienter.

À l'encontre de cette lecture qui voit en l'homme la source de son propre mouvement mais qui lui conserve la possibilité d'une subordination active, la célèbre image de la marionnette ou du jouet divin évoquée par deux fois dans les Lois $^{42}$ peut faire croire à une forme de détermination mécanique de sa conduite: les dieux tireraient dans les coulisses les ficelles de ce pantin. En réalité, l'image n'a pas exactement ce sens. Outre qu'entendue de la sorte, elle contredirait formellement son enseignement constant sur l'âme automotrice, Platon prend bien soin de ne pas imputer directement aux dieux la conduite des hommes. Sa thèse sur la providence divine, qui met en exergue un certain ordre de la nature

39 "Chaque partie du tout, autant qu'il est possible, fait et subit ce qu'il convient qu'elle fasse et subisse" (Lois, X 903b4-5).

40 "Lâme subit des changements, soit sous l'effet de sa propre activité, soit sous celui d'une autre âme» (Lois, X 903d5). Sur l'illimitation du vivant, voir Philèbe, 32b1.

41 L'homme est un animal mimétique (voir 785b). L'Athénien fait fond sur cette aptitude humaine dans le type d'éducation «musicale» qu'il propose au livre II des Lois, tandis que la pratique politique est conçue dans la cité de Magnésie comme l'imitation du gouvernement des dieux par la loi rationnelle (713e6).

42 Lois, I 644d8, VII 803d5. 
et une forme de justice immanente ${ }^{43}$, évacue toute intervention locale ou tout jugement ad hoc. De manière générale, les Lois se gardent bien de faire parler les dieux et les tiennent en retrait des affaires humaines.

Que voudrait alors suggérer l'image d'un théâtre de marionnettes? Moins l'idée que les dieux manipuleraient directement les hommes que celle selon laquelle ils en seraient les spectateurs amusés: c'est que les hommes ressemblent aux dieux comme les marionnettes ressemblent aux hommes. Et tout comme les hommes se plaisent au spectacle de marionnettes, de même les dieux se plaisent au spectacle des hommes.

L'image souligne aussi, bien sûr, qu'hommes et marionnettes ont en commun de ne pas exercer de souveraineté pleine et entière sur leurs mouvements: les gestes des uns et des autres sont le résultat de tractions exercées sur des fils invisibles. Ces fils, pour ce qui concerne les hommes, sont ceux du plaisir et de la peur: la pensée et la raison ne contribuent le plus souvent à leurs déplacements que de manière instrumentale. Parfois cependant la commande d'or interne (645a7), celle du logos, prévaut. Les hommes sortent alors de la caricature. À défaut d'égaler les maîtres du cosmos, ils s'en approchent avec liberté et peuvent prendre leur part du jeu divin ${ }^{44}$.

Contrairement donc à ce que laisse croire une lecture hâtive du mythe des marionnettes, les dieux ne sont pas tout-puissants ni ne dispensent les hommes de se conduire par eux-mêmes (jamais il n'est dit qu'ils les font mal agir, mal penser, mal juger...). À l'inverse, par leur parenté naturelle avec eux, comme par le gouvernement du monde dont ils s'acquittent, les dieux offrent aux hommes un modèle de ce que pourrait être le gouvernement de soi ou de la cité.

43 L'Athénien combine d'ailleurs le changement moral autonome (904b8-c3, voir aussi 785a5-c1) à un mécanisme naturel d'élévation ou de descente dans la hiérarchie des lieux célestes, assurant la justice divine. T. J. SAunders ("Penology and Eschatology in Plato's Timaeus and Laws», p. 240) souligne l'originalité physique de cette providence, qui repose sur un processus automatique dispensant de tout tribunal et de tout jugement, de tout lieu et de tout temps spécifiques.

44 Voir Lois, X 903d5: la justice divine, qui fait allouer par une sorte de loi naturelle des places différentes dans le cosmos aux âmes en fonction de leurs initiatives morales, est comparée à un jeu de pions déplacés sur un échiquier ou un plateau. Voir, sur le terme $\pi \varepsilon \tau \tau \varepsilon v \tau \varepsilon \dot{\varepsilon}$, R. G. Austin, "Greek Board Games», p. 257-271, et sur ce passage des Lois, N. Powers, «Plato's Cure for Impiety in Laws $10 »$, p. 61. 
Cette imitation est d'autant plus requise qu'à la continuité s'ajoute l'inclusion. L'homme ne partage pas seulement avec le divin l'automotricité, son propre mouvement est inclus dans celui du ciel. Cette inclusion est aussi une antériorité: la conduite des dieux précède celle des hommes, le monde accomplit ses révolutions avant et pendant que l'homme élabore sa conduite. Bien sûr, comme il a été établi, la priorité temporelle et causale est d'abord celle du principe automoteur, lequel n'est pas en tant que tel rationnel. En revanche, il est vraisemblable (à défaut d'être démontré) que l'âme associée à l'intellect dirige les mouvements cosmiques ${ }^{45}$.

L'Étranger d'Athènes, on s'en souvient, dénonçait l'erreur fondamentale des esprits forts, qui ne voyaient dans l'art humain qu'un pâle et lointain reflet d'une nature que caractérisent la puissance et le hasard. C'est en faveur d'un renversement complet de cette hiérarchie qu'il plaide tout le long du livre $\mathrm{X}$ des Lois. L'homme ne doit pas revenir à une nature aveugle et brutale, laquelle n'existe pas, mais tâcher dans sa conduite de se rendre digne du grand art des dieux, qui le précède et l'enveloppe. La technè des dieux est donc à l'horizon de sa propre technè. Reste à en comprendre exactement le sens.

L'être humain est plongé dans un univers dont la rationalité n'est pas une donnée, un ordre trouvé là, ou simplement découvert, mais un ordre qu'il sait construit, un ordre élaboré et produit, un ordre rapporté à un agent qui l'a conçu et imposé par son intelligence. De sorte que cette précédence et cet enveloppement de l'art divin incitent l'agent intramondain à reproduire non pas l'ordre du ciel, mais la production de l'ordre. Il s'agit moins pour lui de transposer des lois ou des dispositions cosmiques à la cité (chose qui n'a pas grand sens) que de fabriquer par lui-même un ensemble de règles et de limites qui vaillent pour les corps dont il a la charge, à l'instar de ce que font les dieux pour le ciel. Parce

45 L'argument développé par l'Athénien en faveur de la providence (les dieux ont autant d'aisance à se soucier de détail du cosmos que de son allure générale), contraste vivement avec la description de l'histoire de l'humanité et le mythe de Cronos développés aux livres III et IV. Ces deux derniers textes font une large part, dans le monde humain, à la contingence et à la chance. Sur ce contraste, voir les éclairages différents de A. W. Nightingale, "Historiography and Cosmology in Plato's Laws", p. 299-325, et L. M. Trelawny-Cassity, "On the Foundation of Theology in Plato's Laws", p. 325-349. 
qu'il est inséré dans un monde ordonné par l'intelligence, l'homme est invité par le dieu à se faire l'architecte intelligent de sa vie et de sa cité.

Associer en outre l'intelligence au mouvement circulaire, dans le cas des dieux qui gouvernent le monde, c'est proposer la figure de l'indépendance, de l'autarcie, de l'autonomie, de la clôture sur soi, comme guide de la conduite humaine. Comment en effet les dieux se comportent-ils? En ne dépendant que d'eux-mêmes, en se rapportant le plus possible à eux-mêmes, en conservant les mêmes rapports. Comment les hommes doivent-ils se comporter s'ils veulent imiter les dieux? En étant rationnels et autonomes, en se tenant dans les bornes que fixent à leur conduite leur droit et leurs institutions. Ils doivent faire preuve de cohérence et de précision dans les diverses formes de distribution et de répartition auxquelles procède la cité (par exemple des terres, des fonctions et des magistratures, des châtiments en cas d'infraction), ils doivent borner et clôturer le monde de la cité (le territoire, la population, la richesse), exactement comme les dieux limitent et encerclent le cosmos.

On voit donc la leçon paradoxale: celle d'une autonomie qui refuse la croyance en sa propre auto-institution, puisqu'elle se pose comme dérivée d'un ordre dans lequel l'humain se trouve inclus. Inclusion en vertu de laquelle la partie se trouve enjointe par le tout d'être source de sa propre organisation, conformément à sa nature d'être automoteur, et selon les réquisits d'une raison comparable à celle des dieux. L'homme, invité par l'intelligence cosmique à s'administrer lui-même et à administrer par lui-même sa polis, imite son modèle en respectant des critères essentiellement formels: cohérence, précision, unité et clôture. Il appartient à l'animal rationnel de s'imposer des limites, de cultiver cet art de la mesure qu'illustre à son tour la fondation de la cité des Magnètes.

Il y a bien une nouveauté par rapport à la cosmologie du Philèbe (et à celle du Timée) : en insistant de façon plus marquée sur l'automotricité de l'âme, Platon met davantage l'accent sur la liberté humaine, laquelle est cependant un pur principe de commencement, susceptible de prendre autant une direction qu'une autre. Que l'âme humaine ne puisse être mue mécaniquement par d'autres âmes, aussi divines soient-elles, n'en fait pas pour autant, même si c'est là une condition de possibilité, un centre animé d'un souci de soi, capable de s'interroger et de décider pour soi. Cette réflexivité n'est pleinement possible que grâce à une disposition supplémentaire, inégalement répartie parmi les individus, 
celle de l'intellect. Le pli réflexif et directif de cette faculté découle de la situation faite à l'homme: d'une part, l'absence de dieux locaux et d'une providence particulière qui dirigerait les individus pas à pas, d'autre part, la présence enveloppante d'un grand modèle, d'un monde qui s'autogouverne rationnellement. Aussi l'exercice de cette liberté éclairée et responsable, qui vise à la maitrise rationnelle de soi-même, ne s'impose-t-il que du fait de la reconnaissance de cette première dépendance.

Un trait remarquable de l'autodétermination cosmique est la constance et la permanence. Faute de cet exemple normatif, les hommes ne respecteraient plus leur passé, leurs anciens et leurs législateurs, alors que c'est dans la fidélité à l'héritage reçu que se manifeste la plus grande cohésion collective et temporelle dont ils sont capables. De sorte qu'à l'intérieur de la cité des Magnètes, compte tenu de la variété et de la faiblesse de l'intelligence en la plupart des hommes, ce n'est ni la libre discussion, ni la pleine persuasion rationnelle, mais un mélange d'éducation musicale et corporelle, d'enseignement et de châtiments, qui garantit la persévérance et la régularité de leur conduite ${ }^{46}$.

Voilà pourquoi il importe de bannir les athées. Non seulement ils ne sauraient éviter l'incohérence des choix, voire le choix de l'incohérence ou celui du non-choix, mais encore, ils entretiendraient, volens nolens, le mythe d'une liberté qui serait à elle-même son fondement. L'existence des dieux n'est, de ce point de vue, que l'autre nom pour dire la nature seconde et dérivée de l'autonomie des hommes.

\section{Conclusion : la fabrication de la fabrication du monde}

Récapitulons, pour terminer, notre réponse à la question principale que fait naître le passage cosmologico-théologique des Lois: à quoi peuvent bien servir des dieux dont l'existence reste rationnellement indécise et dont l'intervention traditionnelle dans les affaires humaines est écartée?

La réponse est qu'ils constituent moins une réalité préexistante qu'un paradigme. Ce paradigme est l'œuvre d'une intelligence philosophique qui élabore l'image vraisemblable d'un monde habité par l'intelligence divine.

46 Voir ici R. MaYhew («Persuasion and Compulsion», p. 108-111), qui souligne la nature instable et contradictoire de ce mélange. 
Qu'il s'agisse d'une construction, l'Athénien l'indique de deux manières différentes. La première mentionne son habileté à former des images. La seconde tient tout simplement à ce qu'il ne fait guère de cas de l'observation des faits; contrairement à l'apparence, il ne produit pas une preuve à partir des effets, mais il propose un modèle a priori du monde, conformément d'ailleurs à la fonction que Platon assignait à l'astronomie dans la République.

Lorsqu'il s'agit de fixer la nature du mouvement cosmique et de reconnaître l'âme à laquelle il convient de le rattacher, l'Athénien met les phénomènes hors jeu, du moins ceux qui permettraient d'identifier à coup sûr le mouvement de l'intellect. Celui-ci, s'il existe, ne se laisse pas saisir directement. On ne peut l'appréhender qu'au moyen d'une «image», le mouvement circulaire, et c'est par rapport à ce mouvement circulaire idéal que se décidera la nature du mouvement du monde. L'Athénien prend soin de préciser qu'il a fabriqué cette "belle» image à l'imitation du mouvement du potier, démiurge par excellence. Mais qu'a-t-il fabriqué précisément dont il puisse être si fier? Ce ne peutêtre la comparaison entre les deux mouvements, celui du tour et celui $\mathrm{du}$ cercle, qui tombe sous le sens. C'est bien plutôt l'assimilation du mouvement de l'intellect à un mouvement circulaire, et, par extension, celle du mouvement du monde au mouvement circulaire. Il s'agit d'un montage ou d'une "élévation", comparable à l'opération par laquelle le potier lève la glaise et lui donne forme et volume, ou à celle du menuisier qui arrondit la boule de bois.

De fait, le rapprochement opéré entre ces deux mouvements du cercle et de l'intelligence, dont les différences sont délibérément laissées dans l'obscurité par l'Athénien, conduit naturellement, dans un deuxième temps, à tenir pour négligeables les écarts que l'on pourrait détecter dans la nature entre le ciel et ce modèle de rotation circulaire. Quand vient le moment fatidique de savoir quels sont les mouvements des astres, nul appel à l'observation, nulle donnée empirique collectée, mais un acte de foi: des deux hypothèses, celle selon laquelle la marche du monde est régulière et de nature similaire au mouvement imagé de l'intellect, ou celle selon laquelle elle serait désordonnée et folle (alternative en ellemême bien simplificatrice, puisque nous avons vu que nous pouvions concevoir a priori toutes sortes de mouvements intermédiaires), l'une est exclue et l'autre est élue sur la base de la seule piété de Clinias. 
Le choix de l'uniformité et de la circularité du mouvement de l'univers découle moins du constat d'une réalité extérieure qu'il n'exprime la qualité de l'âme sommée de choisirir ${ }^{47}$. Eût-il d'ailleurs fallu établir empiriquement la circularité parfaite des révolutions astrales que l'Athénien aurait été dans l'embarras: n'est-elle pas partiellement démentie par le mouvement rétrograde des planètes (821b8-10)? Et le "problème de Platon» tel que le rapporte Simplicius ("quels sont les mouvements circulaires, uniformes et parfaitement réguliers qu'il convient de prendre pour hypothèses, afin que l'on puisse sauver les apparences présentées par les planètes? ${ }^{48}$ ) n'a pas reçu de solution géométrique satisfaisante dans les Lois.

Dans le Timée, en revanche, Platon a suggéré que le mouvement résultant de la composition de deux mouvements circulaires uniformes d'axes inclinés l'un par rapport à l'autre est un mouvement en spirale (39a1-b1). Voilà qui serait susceptible de décrire sommairement le mouvement de la lune, du soleil et des planètes par rapport au Zodiaque. Un passage des Lois (VII, 822a-b) paraît y faire allusion, en indiquant du reste que c'est là un point que l'Athénien (Platon?) n'apprit (ou ne découvrit) que tardivement (821b4). Néanmoins, pour rendre raison des rétrogradations et stations apparentes des cinq planètes (dont sont dépourvus le soleil et la lune), il faut le modèle complexe de plus de 20 sphères homocentriques d'Eudoxe, dont Platon ne fait jamais état ${ }^{49}$.

47 Voir dans le Sophiste (265d-e) un mouvement parallèle, qui fonde sur une disposition de l'âme de l'interlocuteur (son naturel) le type de nature (ordonné et divin) attendu dans le cosmos. Même mention de la "piété» (de Protarque) quand il s'agit de décider de l'ordre de l'univers dans le Philèbe (28e2), ordre conçu comme l'œuvre du voṽ s par des sages qui trouvent ainsi motif à se flatter (à glorifier l'intelligence dont ils sont si fiers, Éavtoùc

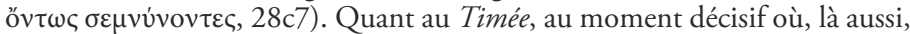
il s'agit de trancher entre deux hypothèses, celle d'un monde beau et d'un démiurge bon, et celle qui lui est opposée, la loi sacrée interdit d'évoquer

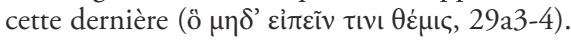

48 Simplicius, In Aristotelis quatuor libros de Coelo commentaria, p. 488 et p. 493.

49 Sur les insuffisances de la «solution de Platon", voir l'étude classique de P. Duhem, Le Système du monde, p. 107-111; en dernier lieu, B. VitraC, «Les mathématiques dans le Timée de Platon: le point de vue d'un historien des sciences", p. 11-78. 
En somme, il ne s'agit pas, dans les Lois, de dire ce qu'est le monde, mais de dire à quoi il doit ressembler. Et ce à quoi il ressemble se laisse déterminer a priori ${ }^{50}$. Au sein de cette image, les hommes sont eux-mêmes inclus et trouvent dans la conduite des dieux astraux un modèle à leur propre conduite ("dieu est la mesure», ou l'étalon, des vertus humaines, IV, 714c4). Le gouvernement du monde par lui-même devient la raison d'être du gouvernement de soi par les hommes, libres dans leur subordination même.

Existe-t-il un référent à cette image cosmologique? Si référent il y a, ce n'est pas un dieu existant en soi et par soi ${ }^{51}$, mais une essence, la même que celle qui inspira la libre élaboration de la kallipolis dans la République. Avec les dieux et avec le cosmos, comme avec la cité, nous n'avons pas affaire à des réalités intelligibles, mais, dans le meilleur des cas, à des paradigmes engendrés à partir d'une réalité intelligible entendue comme l'ensemble des rapports formels et hiérarchiques constitutifs de l'idée de tout organisé (idée de justice). Il se pourrait bien, après tout, que la raison du philosophe législateur soit seule avec elle-même et que les lois qu'elle établit pour la cité partagent cette intelligence ${ }^{52}$ qu'elle suppute généreusement au cœur de l'univers.

\section{Bibliographie}

Aвolafia Jacob, "Solar Theology and Civil Religion in Plato's Laws", Polis, 32, 2015, p. 369-392.

Austin Roland, "Greek Board Games», Antiquity, 14, 1940, p. 257-271. Bовоnich Christopher, "Persuasion, Compulsion, and Freedom in Plato's Laws", Classical Quarterly, 41, 1991, p. 365-87; repr. in FIne Gail (ed.), Plato: Ethics, Politics, Religion, and the Soul, Oxford: Oxford University Press, 2000, p. 373-403.

50 Évidemment, il convient de faire en sorte que cette ressemblance soit vraisemblable. D'où le programme de recherche fixé à l'Académie.

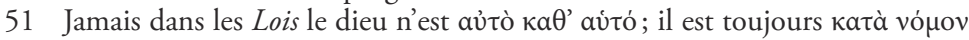
(X 890a5-6, 891e2, 904a9). Pour d'autres références et une discussion générale, voir F. Teisserenc, «Le dieu de la loi», p. 66.

52 «Nous devons obéir à tout ce qui en nous participe de l'immortalité, donnant le nom de loi [vónov] à la distribution de l'intelligence [voṽ

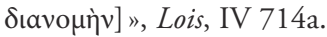


Brill Sara, «Psychologie and Legislation in Plato's Laws», Proceedings of the Boston Area Colloquium in Ancient Philosophy, vol. 26, $\mathrm{n}^{\circ}$ 1, 2011, p. 211-251, p. 221-224.

Platon, Les Lois, Livres VII à XII, traduction inédite, introduction et notes par Luc Brisson et Jean-François Pradeau, Paris: GF Flammarion, 2006.

Dunem Pierre, Le Système du monde, vol. 1, Paris: Hermann, 1913.

GuÉroult Martial, «Le Xe livre des Lois et la physique platonicienne», Revue des Études grecques, 37, 1924, p. 27-78.

Mayhew Richard, "Persuasion and Compulsion in Plato's Laws 10 ", Polis, 24, $\mathrm{n}^{\circ}$ 1, 2007, p. 91-111.

Menn Stephen, Plato's God as Nous, Carbondale: Southern Illinois University Press, 1995.

Nightingale Andrea, "Historiography and Cosmology in Plato's Laws", Ancient Philosophy, 19, n 2, 1999, p. 299-325.

Powers Nathan, "Plato's Cure of Impiety in Laws X», Ancient Philosophy, 34, 2014, p. 47-64.

Quine Willard, Le Mot et la Chose, trad. fr. J. Dopp et P. Gochet, Paris: Flammarion, 1977 [1960].

Saunders Trevor, "Penology and Eschatology in Plato's Timaeus and Laws», Classical Quarterly, 23, n 2, 1973, p. 232-244.

Simplicius, In Aristotelis quatuor libros de Coelo commentaria, ed. Heiberg J.-L., Berolini: Typ. et impensis G. Reimeri, 1894.

Teisserenc Fulcran, "Le dieu de la loi», Philosophie antique, 18, 2018, p. 31-69.

Teisserenc Fulcran, "D'une causalité démiurgique en cosmologie et en éthique", in: Dillon John and Brisson Luc (eds.), Plato's Philebus, Selected Papers from the Height Symposium Platonicum, Sankt Augustin: Academia Verlag, 2010, p. 342-347.

Teisserenc Fulcran, "Puissance, activité et passivité dans le Sophiste», Philosophie, 96, 2007, p. 25-45.

Trelawny-Cassity Lewis, "On the Foundation of Theology in Plato's Laws", Epoché, 18, n² 2, 2014, p. 325-349.

Vitrac Bernard, "Les mathématiques dans le Timée de Platon: le point de vue d'un historien des sciences", Études platoniciennes, 2, 2006, p. 11-78. 